\title{
NUMERICAL EVOLUTIONARY CORRELATION
}

LESS, György, Geological Institute of Hungary, H-1442 Budapest, P.O.Box 106, Hungary; Ó.KOVÁCS*, Lajos, Hungarian Geological Survey, H-1440 Budapest, P.O.Box 17, Hungary

NUMERICAL EVOLUTIONARY CORRELATION correlates the development of fossil evolutionary lineages by capitalizing on their co-occurrence in particular samples and establishes numerical (num.) evolutionary (ev.) scales. The age of fossil-assemblages can be estimated in years, so this method of correlation can be applied to any field of geology. Based on a biometrical and population-statistics background, the age-determination is objective and controllable.

A NUMERICAL EVOLUTIONARY SCALE is a framework calibrated by the net ev. effect extracted from the network of ev. lineages. In a num. ev. scale particular fossil-assemblages coming from specific localities occupy their position proportionally to their net ev. grade. Using the scale as an intermediate language, ev. lineages can be directly correlated with each other. Reflecting ev. proportions, the scale is independent of num. time scales, but it is correlatable with any of them using tie-points. Moreover, it can be one of the most regular reflections of geological time. Ev. ages (ev. grades identified in two points with a geological time scale) can be converted into num. geological ages and these in turn can be used for calibrating num. time scales. The unit of measurement for the scale corresponds not to the time itself, but its ev. reflection, and is defined as ev. million year = Mega annum evolutionare (Mae.).

PRINCIPLE: Ev. lineages are considered to be ev. clocks that show ev. age through a precisely defined and objectively measurable parameter (or its mathematically transformed form), whose linear relation to the time can be observed or supposed. By testing simultaneously these clocks, their irregularities and inaccuracies can be balanced by one another using an iterative procedure: First we estimate the ev. age functions (regression lines between the ages and the parameter values), then we make more and more precise estimates for the ev. ages of the samples and for the ev. age functions, alternatively. Our experiments (supported by simulation test series) show that using realistic starting estimates, the iterative procedure is not only convergent (and in this case independent of the starting estimates, too), but open as well, i.e. it can incorporate any time-reflecting data-series and, therefore, particular num. ev. scales can be integrated later.

ADVANTAGES: 1 . Paleontological determinations become simpler. They are reduced to separating populations within a given sample, identifying their ev. lineage and measuring/counting a num. parameter for each specimen. 2. The age of the sample may be determined by substituting into the previously computed ev. age-functions without repeating the basic iterative procedure. This process is objective and can be controlled. 3. The error of these ev. ages can be estimated. It reflects the contradiction between the ages obtained from the different populations belonging to the given sample.

APPLICATION: The procedure has been applied to $20 \mathrm{ev}$. lineages of European Orthophragminae (larger Foraminifera) using 51 European samples. Considering its internal proportions, the obtained num. ev. scale is compatible with those of num. time-scales; therefore, it is suitable for geological age-determination as well. The corresponding paper is in press in Geobios (Lyon, France).

ACKNOWLEDGEMENTS: This research was partly financed by the National Scientific Research Fund of Hungary (OTKA, Grant No. T 16863). 